\title{
Approche illustrée des bases de l'électronique
}

\author{
Yves BERNARD \\ bernard@lgep.supelec.fr \\ LGEP-Supelec 11 rue Joliot Curie Plateau du Moulon 91192 Gif sur Yvette
}

RESUME Les buzzers piézoélectriques sont utilisés comme un outil pédagogique pour illustrer les notions de base en électronique. Une maquette simple de récupération d'énergie sert de fil conducteur au cours et montre un générateur de courant, les notions d'adaptation d'impédance, de bande passante, de schéma équivalent. Les équations de bases sont tirées de la physique des matériaux et les lois fondamentales de l'électronique sont illustrées sans aborder le traitement du signal électrique qui ne peut rester que basique à ce niveau du cours.

Mots clés : piézoélectricité, récupération d'énergie, schéma équivalent, bases de l'électricité

\section{1}

\section{INTRODUCTION}

Le problème pédagogique auquel l'enseignant d'électronique dans un cursus de physique est de plus en plus confronté est l'intérêt des étudiants pour ce qu'il a à leur dire. Cet aspect est d'autant plus développé lorsqu'il s'agit d'enseigner les bases de la matière puisqu'alors il est très difficile d'ouvrir le cours sur les applications alors que l'auditoire n'a pas les connaissances et le vocabulaire nécessaires. Une manière de contourner cet aspect est de montrer ce que l'électronique peut apporter aux autres matières et les analogies qui peuvent exister.

Cet article évoque l'utilisation d'un buzzer piézoélectrique comme source. L'objectif étant au travers de l'explication du fonctionnement de cet objet d'aboutir à une illustration de notions de base de l'électronique. Cette expérience pédagogique est tentée cette année pour la première fois sur des étudiants de L2 physique. Un retour sur expérience pourra être fait au moment du CETSIS.

Dans cet article, on ne rappellera pas les notions de base de la piézoélectricité mais il semble indispensable de le faire auprès des étudiants qui ont besoin comme pré requis des notions de base de l'électrostatique et de la mécanique (qu'est ce qu'un champ électrique, une contrainte ?). On utilisera l'effet piézoélectrique direct pour créer un générateur et l'effet piézoélectrique inverse afin par exemple d'illustrer ce qu'est une résonnance.

Nous travaillons en cours intégré (cours, TD, TP), l'idée est de présenter la manipulation lors de la première séance (durant $1 / 2$ heure) et d'y refaire appel régulièrement comme illustration lors des autres séances.

\section{MISE EN PLACE DES EQUATIONS DU BUZ-} ZER

L'échantillon utilisé comme démonstrateur est un buzzer de la société Murata ${ }^{\circledR}$ commercialisé sous la dénomination Ceramitone ${ }^{\circledR}$. Il se trouve facilement [1] et son coût est inférieur à 5 euros. Il est constitué d'une armature en résine qui contient une pastille de céramique piézoélectrique utilisée en flexion [2].

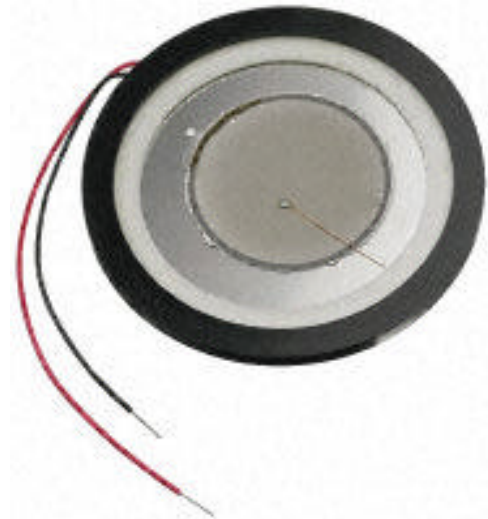

fig 1 : Echantillon piézoélectrique utilisé

\subsection{A des fréquences inférieures à la première ré- sonnance}

Dans cette partie, on cherche à écrire les équations régissant le fonctionnement en générateur (effet direct).

En quasi statique, les équations de la piézoélectricité lient 2 grandeurs électriques ( $E$ champs électrique et $\mathrm{D}$ induction électrique) et 2 grandeurs mécaniques ( $\mathrm{S}$ déformation et $\mathrm{T}$ contrainte). En sollicitant le matériau en deçà de sa première fréquence de résonnance (on montrera par la suite qu'elle se situe à $525 \mathrm{~Hz}$ ), ces équations permettent de calculer une grandeur électrique ou mécanique à partir de 2 autres et des conditions aux limites. L'exemple qui sera utilisé par la suite est l'équation liant l'induction électrique $\mathrm{D}$ à la contrainte appliquée au matériau $\mathrm{T}$ et au champ électrique E auquel il est soumis.

$$
D=d^{E} T+\varepsilon^{T} E
$$

En dérivant par rapport au temps, on obtient l'équation 2

$$
\frac{d D}{d t}=d^{E} \times \frac{d T}{d t}+\varepsilon^{T} \times \frac{d E}{d t}
$$

On supposera que la contrainte appliquée lorsqu'on exerce une force sur la pastille piézoélectrique est uniquement de flexion ainsi le problème devient scalaire. $\mathrm{E}$ est l'image de la tension $\mathrm{V}$ aux bornes du buzzer 
( $E=V / e$ où e est l'épaisseur de la pastille). De la même manière $\mathrm{D}$ est l'image de la quantité de charges $\mathrm{Q}$ sur les électrodes ( $D=Q / A$ où $A$ est la surface de la pastille). Avec $\mathrm{T}=\mathrm{F} / \mathrm{A}$ (où $\mathrm{F}$ est la force appliquée à la membrane), cette équation s'identifie à

$$
i=i_{o}-C_{p} \frac{d V}{d t}
$$

Avec $i_{o}=d^{E} \times \frac{d F}{d t}$ et $C p=\frac{A \varepsilon^{T}}{h}$

On aboutit donc au schéma équivalent de la figure 2 .

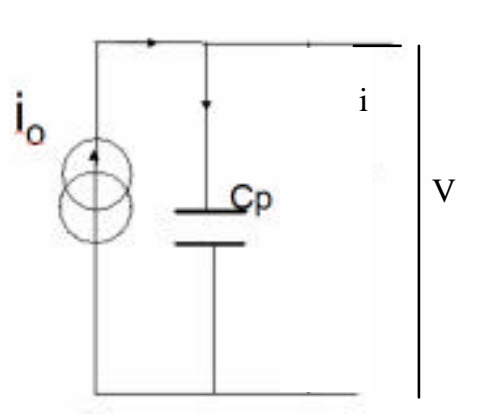

fig 2 : Schéma équivalent du dispositif sollicité mécaniquement

On précise que dans ce schéma les pertes diélectriques sont négligées.

\subsection{Autour des fréquences de résonances}

Dans cette partie, on cherche à écrire les équations régissant le fonctionnement en récepteur (effet inverse).

Les matériaux piézoélectriques sont des diélectriques. L'application du champ électrique dans lequel est plongé le matériau se fait par le biais de deux électrodes déposées sur le matériau. Du point de vue électrique, l'échantillon ainsi constitué (matériau + électrodes) est un condensateur. Son impédance est donc constituée d'un condensateur de capacité $\mathrm{C}_{0}$ (qui n'est autre que $G_{\mathrm{P}}$ ) en parallèle avec une résistance $R_{0}$ qui traduit les pertes diélectriques (négligées précédemment).

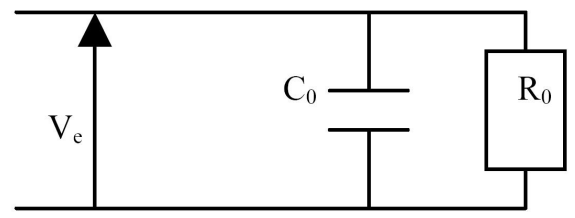

fig 3 : Charge électrique équivalente en statique

La capacité $\mathrm{C}_{0} \mathrm{~s}$ 'écrit $\mathrm{C}_{0}=\varepsilon \mathrm{A} / \mathrm{e}$ où $\varepsilon$ est la permittivité électrique du matériau. La résistance $R_{0}$ se calcule avec la formule $R_{0}=\rho e / A$ où $\rho$ est la résistivité du matériau.

Cependant, le générateur qui alimente cet échantillon fournit, en supplément de la puissance électrique mise en jeu dans le condensateur, une puissance mécanique qui induit les déformations subies par l'échantillon. Les efforts mis en jeu sont de trois natures : une force de rappel $\left(\mathrm{F}_{\mathrm{r}}\right)$, liée au comportement élastique du matériau, une force d'inertie $\left(\mathrm{F}_{\mathrm{i}}\right)$ liée au caractère dynamique de la sollicitation et une force de frottement $\left(\mathrm{F}_{\mathrm{f}}\right)$ qui traduit les pertes mécaniques. En notant $\mathrm{u}$ la vitesse de déplacement des faces lorsque celles ci sont libres, on obtient $\mathrm{F}_{\mathrm{f}}=\mathrm{fu}$ (f coefficient de frottement $), \mathrm{F}_{\mathrm{i}}=\mathrm{M}$ ? $\mathrm{u} / \mathrm{t}$ ( $\mathrm{M}$ coefficient d'inertie), $\mathrm{F}_{\mathrm{r}}=\mathrm{k}$ ? $\mathrm{u}$ ?t (k coefficient de raideur). Ces trois forces s'additionnent et peuvent se représenter avec une analogie électrique mécanique sous forme d'un schéma électrique où les forces sont représentées par des tensions et les vitesses par des courants.

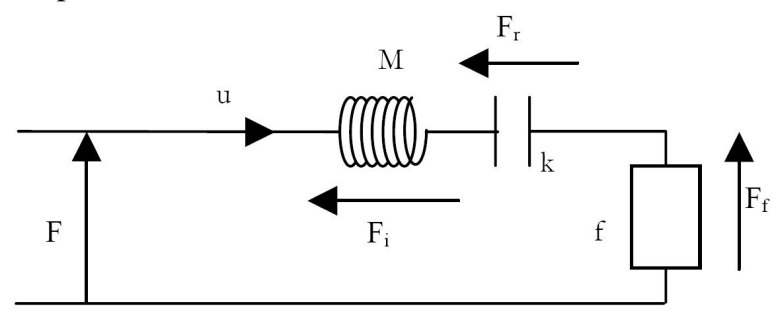

Fig.4 : Schéma électrique représentant le comportement mécanique de l'échantillon en dynamique.

La puissance instantanée fournie par le générateur est la somme de la puissance électrique reçue par le condensateur équivalent et de la puissance mécanique acquise par le matériau. En tenant compte du couplage électrique et mécanique, on aboutit au schéma équivalent donné sur la figure 4 où le coefficient $\mathrm{N}$ est un coefficient piézoélectrique qui transforme la tension aux bornes de l'échantillon en force et le courant dans l'échantillon en vitesse de déplacement.

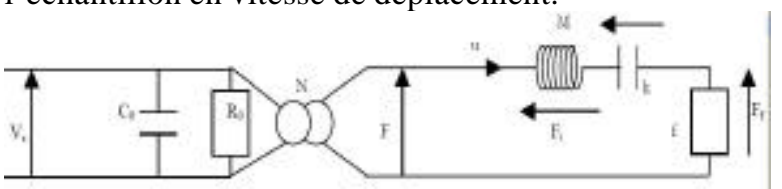

Fig.5 : Schéma électrique représentant le comportement électromécanique de l'échantillon en dynamique.

On peut alors ramener le secondaire du transformateur au primaire moyennant le changement de variable $\mathrm{L}=\mathrm{MN}^{2}, \mathrm{C}=\mathrm{k} / \mathrm{N}^{2}, \mathrm{R}=\mathrm{fN}^{2}$. On obtient le schéma de Masson (figure 6) dans lequel la branche R, L, C est dite motionnelle.

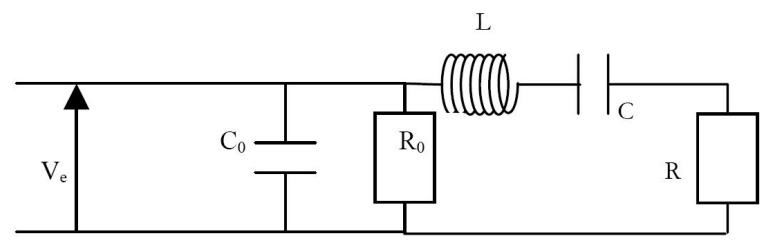

Fig.6 : Schéma de Masson.

\section{UTILISATION DU DISPOSITIF}

Les équations relatives à différents tests sur le buzzer ayant été mises en place, il reste à exploiter habilement ces phénomènes physiques dans le cadre d'un 
cours sur les bases de 1'électronique. On cherchera à illustrer les notions de générateur, les aspects de régime temporel avec la décharge d'un condensateur et la notion d'impédance équivalente en faisant remarquer les résonnances. Le son émis par le buzzer est supportable pour peu que la tension d'alimentation soit faible, en outre cela permet de bien illustrer la relation fréquence/note.

\subsection{Générateur de courant}

On pourra bien sur ici utiliser les équations (1) à (3) pour obtenir le générateur de tension équivalent mais conserver la représentation sous la forme du générateur de courant peut servir à illustrer une notion qui passe difficilement auprès des étudiants.

Dans un premier temps, on peut percuter manuellement la pastille afin d'observer la tension de sortie à l'oscilloscope. Le signal étant fugace, la sollicitation mécanique peut être effectuée à l'aide d'un motoréducteur sur lequel on place une roue crantée (figure 7).

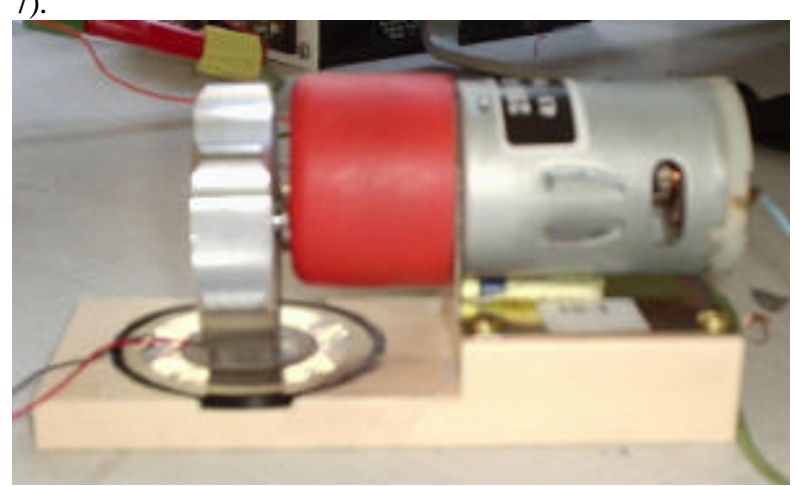

fig 7 : Motorisation de la sollicitation mécanique.

On peut alors observer à l'oscilloscope en mesurant la tension aux bornes de différentes résistances le résultat du pont diviseur de courant. La figure 8 montre la comparaison mesure/calcul du courant généré en $\mu \mathrm{A}$ en fonction de la résistance de charge.

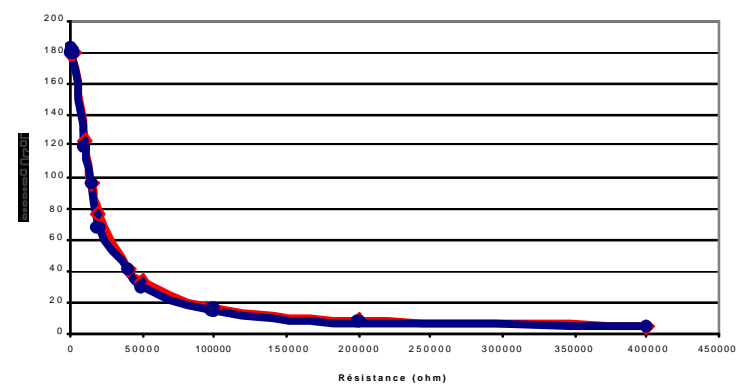

fig 8: Illustration du pont diviseur de courant.

Il peut aussi être intéressant d'observer pour une charge donnée l'effet de la fréquence en faisant tourner plus ou moins vite le moteur.

Cependant, afin d'exploiter complètement ces mesures, il faut connaître les valeurs de $C_{P}$ et $R_{P}$.

\subsection{Etude temporelle}

Afin de caractériser les valeurs de $C_{P}$ et $R_{P}$, on stoppe toute sollicitation mécanique du buzzer. On alimente $\mathrm{C}_{\mathrm{P}}$ en continu de manière à la charger et on observe sa décharge au cours du temps. La réponse est un premier ordre avec une constante de temps de 4,2s.

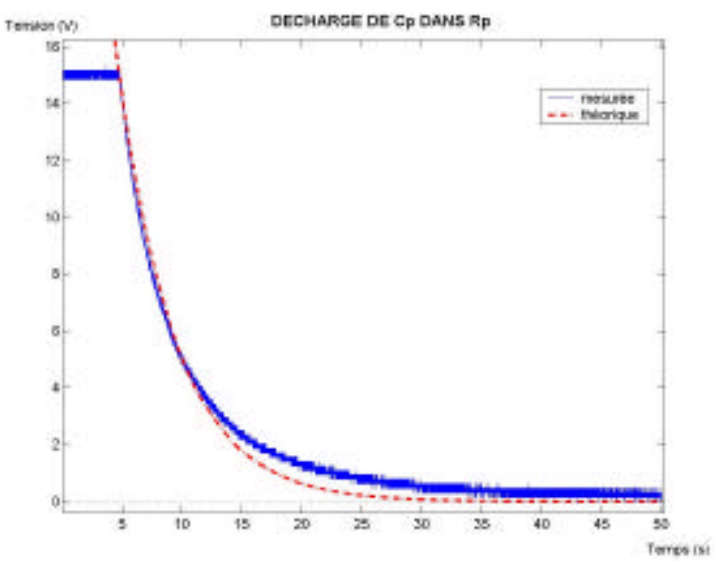

fig 9: Décharge de $C_{P}$ observée à l'oscilloscope

La résistance $R_{P}$ étant bien plus grande que celle d'entrée de l'oscilloscope, on en déduit $\mathrm{C}_{\mathrm{P}}$ de l'ordre de $420 \mathrm{nF}$ ce qui est dans la fourchette donnée par le constructeur de $340 \pm 35 \% \mathrm{nF}$.

\subsection{Notion de résonnance}

En alimentant le buzzer en tension sinusoïdale, celui-ci vibre à la fréquence à laquelle il est soumis. On fera remarquer que le niveau sonore augmente autour des fréquences de résonnance. Le schéma de Masson est particulièrement bien adapté à la mise en évidence de ce phénomène. En outre, une mesure de l'impédance du buzzer en fonction de la fréquence est faisable. Sur la figure 10, on observe l'évolution de l'admittance d'un échantillon sur une gamme de fréquence couvrant les deux premiers modes de résonnance.

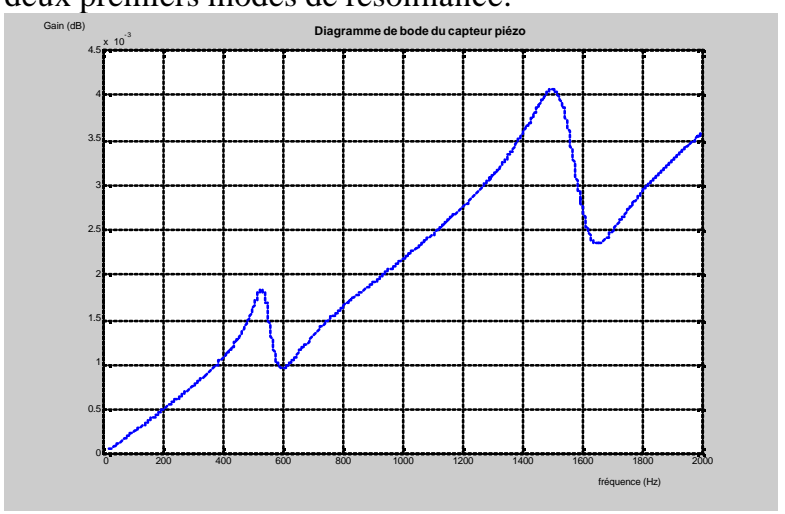

fig 10 : Mesure de l'admittance d'un buzzer.

Il est à préciser que pour modéliser la courbe d'admittance de la figure 10, il faut utiliser deux branches motionnelles dans le schéma de Masson, chacune d'elles étant à même de représenter une et une seule résonnance. 


\section{4}

\section{OUVERTURE VERS DES APPLICATIONS}

Afin d'ajouter un intérêt supplémentaire (au regard des étudiants) à ces démonstrations et à la nécessité de connaître électriquement le dispositif, on peut montrer une manipulation de récupération d'énergie. Si le buzzer est situé dans un environnement soumis à des vibrations mécaniques, on peut utiliser l'énergie électrique qu'il génère à partir de l'énergie mécanique qu'il reçoit.

\subsection{Récupération d'énergie}

Le courant électrique débité par le buzzer peut être redressé et utilisé pour charger un condensateur de stockage (figure 11). A titre d'illustration, on obtient assez rapidement une énergie suffisante pour allumer une LED.

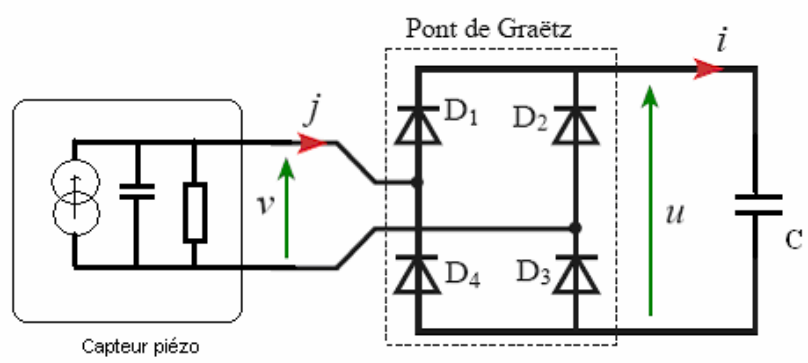

Fig 11 : Circuit de récupération d'énergie

Le principe de récupération d'énergie mécanique à partir de matériaux piézoélectriques trouve déjà des applications dans l'alimentation d'objets nomades autonomes.

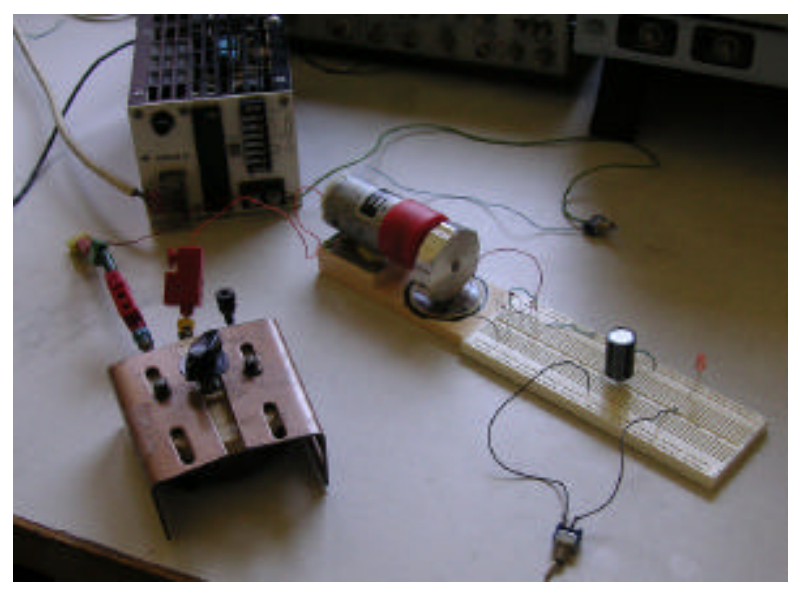

fig 12 : Démonstrateur

\subsection{Autres utilisations possibles du démonstrateur}

Ce dispositif suscite un intérêt chez les étudiants, on peut alors mettre à profit cet intérêt pour les faire travailler sur des sujets touchant d'autres domaines ou plus complexes. Ceci pourra par exemple être fait au niveau M1 dans le cadre de projets courts.

\subsubsection{Dimensionnement du moteur}

Le calcul de dimensionnement du moteur se fait facilement. Il fait appel à des notions de mécanique de base et permet de sensibiliser au choix des actionnements. Pour une roue métallique frottant sur une céramique on pourra prendre un coefficient de frottement de 0,5 . En outre, on peut utiliser le buzzer piézoélectrique comme capteur pour mesurer la force tangentielle qu'exerce la roue sur la pastille.

En supposant que la roue est un cylindre plat plein, son inertie se calcule facilement. Enfin le nombre de dents de la roue et la fréquence maximum de sollicitation mécanique du buzzer permettent de fixer la vitesse de rotation du moteur.

\subsubsection{Adaptation d'impédance}

Un problème bien plus compliqué est celui du choix de la charge intégrant le condensateur de stockage. Afin de sensibiliser les étudiants à ce problème le circuit peut être modélisé par un logiciel de résolution de circuits et on met clairement en évidence que le choix du condensateur de stockage est déterminant.

\section{CONCLUSION}

Un démonstrateur de récupération d'énergie par piézoélectricité a été décrit. Dans le cadre de projets avec des étudiants de niveau L3 ou M1, ce démonstrateur qui trouve la faveur des étudiants permet de se poser d'intéressantes questions.

Cependant c'est surtout comme illustration des bases de l'électronique qu'une partie de ce démonstrateur est utilisé. Différentes expériences simples effectuées sur un buzzer piézoélectrique permettent de faire observer les lois fondamentales de l'électronique sans utiliser de GBF.

On évoque aussi le fait que les circuits électroniques de base peuvent représenter une technique de résolution des équations linéaires.

\section{BIBLIOGRAPHIE}

[1] www.radiospares.com

[2] «Piezoelectric sound components », catalogue No. P37E-20, Murata manufacturing Co.

Je tiens à remercier Ivan ESTEVEZ qui a participé à ce travail dans le cadre de son stage de M1. 